\title{
Planar Matchings for Weighted Straight Skeletons ${ }^{\star}$
}

\author{
Therese Biedl ${ }^{1}$, Stefan Huber ${ }^{2}$, and Peter Palfrader ${ }^{3}$ \\ 1 David R. Cheriton School of Computer Science, University of Waterloo, \\ Waterloo, Ontario N2L 1A2, Canada; biedl@uwaterloo.ca \\ 2 Institute of Science and Technology Austria, \\ 3400 Klosterneuburg, Austria; stefan.huber@ist.ac.at \\ 3 Universität Salzburg, FB Computerwissenschaften, \\ 5020 Salzburg, Austria; palfrader@cosy.sbg.ac.at
}

\begin{abstract}
In this paper, we introduce planar matchings on directed pseudo-line arrangements, which yield a planar set of pseudo-line segments such that only matching-partners are adjacent. By translating the planar matching problem into a corresponding stable roommates problem we show that such matchings always exist.

Using our new framework, we establish, for the first time, a complete, rigorous definition of weighted straight skeletons, which are based on a so-called wavefront propagation process. We present a generalized and unified approach to treat structural changes in the wavefront that focuses on the restoration of weak planarity by finding planar matchings.
\end{abstract}

Keywords: planar matchings, pseudo-line arrangements, stable roommates, weighted straight skeletons

\section{Introduction}

The straight skeleton is a skeletal structure of a polygon $P$, similar to the Voronoi diagram. It was introduced to computational geometry almost two decades ago by Aichholzer et al. [1], and its definition is based on a so-called wavefront propagation process, see Fig. 1: Each edge of $P$ emits a wavefront edge that moves towards the interior of $P$ at unit speed in a self-parallel manner. The polygons formed by these wavefront edges at any given time $t \geq 0$ are the wavefront, denoted by $\mathcal{W}_{P}(t)$, and take the form of a mitered offset of $P$. Over time, the wavefront undergoes two different kinds of topological changes, socalled events, due to self-interference: roughly speaking, an edge event happens when a wavefront edge collapses, and a split event happens when the wavefront splits into parts. The straight skeleton $\mathcal{S}(P)$ of $P$ is then defined as the geometric graph whose edges are the traces of the vertices of $\mathcal{W}_{P}$. Similar to Voronoi diagrams and the medial axis, straight skeletons became a versatile tool for applications in various domains of science and industry [8].

* T. Biedl was supported by NSERC and the Ross and Muriel Cheriton Fellowship.

P. Palfrader was supported by Austrian Science Fund (FWF): P25816-N15. 
The weighted straight skeleton, where wavefront edges do not necessarily move at unit speed, was first mentioned by Eppstein and Erickson [5] and has since been used in a variety of different applications $[2,7,9,10]$. Weighted straight skeletons, with both positive and negative weights, also constitute a theoretical tool to generalize straight skeletons to 3D [3]. Even though weighted straight skeletons have already been applied in both theory and practice, only recently Biedl et al. [4] showed that basic properties of unweighted straight skeletons do not carry over to weighted straight skeletons in general. Biedl et al. [4] also proposed solutions for an ambiguity in the definition of straight skeletons caused by certain edge events and first mentioned by Kelly and Wonka [9] and Huber [8].

In this paper, we discuss another open problem in the definition of weighted straight skeletons caused by split events. An event happens due to a topological change in the wavefront and the event handling was so far guided by one fundamental principle: Between events, the wavefront is a planar collection of wavefront polygons. This is easily achieved when handling edge events and "simple" split events. However, is it always possible to handle multiple simultaneous, co-located split events in a fashion that respects this fundamental

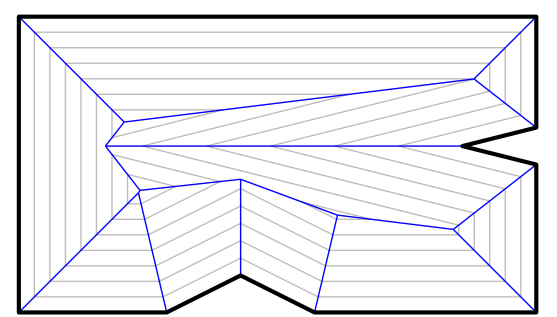

Fig. 1. The straight skeleton $\mathcal{S}(P)$ (blue) of a polygon $P$ (bold) is defined as the traces of wavefront vertices over time. Instances of the wavefront $\mathcal{W}_{P}(t)$ at different times $t$ are shown in gray. principle?

We will show that it is necessary to weaken the requirement of strict planarity in the fundamental principle. After that, we can answer the question to the affirmative and therefore show how to define weighted straight skeletons safely in the presence of multiple simultaneous, co-located split events. (Note that due to the discontinuous character of straight skeletons, it is not possible to tackle this problem by means of simulation of simplicity.) We first rephrase this problem as a planar matching problem of directed pseudo-lines and show how to transform the planar matching problem into a stable roommate problem. For the main result, we prove that our particular stable roommate problem always possesses a solution and those solutions tell us how to do the event handling of the wavefront in order to maintain planarity.

\section{Weighted straight skeletons}

\subsection{The wavefront}

Let $P$ denote a polygon, possibly with holes. We denote by $\sigma(e) \in \mathbb{R} \backslash\{0\}$ the weight of the edge $e$ of $P$ and call $\sigma$ the weight function. For every edge $e$ of $P$, let $n(e)$ denote the normal vector of $e$ that points to the interior of $P$. Initially, every edge of $P$ sends out a wavefront edge with fixed speed $\sigma(e)$. That is, the segments of the wavefront $\mathcal{W}(t)$ at time $t$ that originate from edge $e$ are contained 
in $\bar{e}+t \cdot \sigma(e) \cdot n(e)$, where $\bar{e}$ denotes the supporting line of $e$. If $\sigma(e)$ is negative, the wavefront edge that emanated from $e$ moves to the exterior of $P$.

Intuitively, an event happens when a wavefront vertex meets another wavefront edge or, in particular, another wavefront vertex. The situation becomes more complicated when two or more such events are co-located at the same time $t$. For unweighted straight skeletons, i.e., with all weights set to 1 , the wavefront is planar between events, and we can interpret events as the incidences where planarity is violated. Let us consider the case where multiple wavefront vertices meet at a point $p$. For ordinary straight skeletons, we restore planarity by considering the cyclical order of wavefront edges meeting at $p$ and by re-pairing each edge with a cyclically neighboring edge, see Fig. 2. We call this the standard pairing technique.

In case of weighted straight skeletons, the situation becomes significantly more difficult. First, (strict) planarity cannot always be restored. Second, (weak) planarity may not be restored by the simple pairing scheme mentioned above, and it is not even obvious why some other pairing scheme that restores planarity must exist.

Consider Fig. 3, where two vertices, $u$ and $v$, meet simultaneously at point $p$ and time $t$. By construction, the vertex $v$ lies on the supporting line of $e$ for a positive-length time interval. We show the supporting lines of the edges at time $t+\delta$, with $\delta$ being positive but small. We have three combinatorial possibilities to pair up the wavefront edges. One

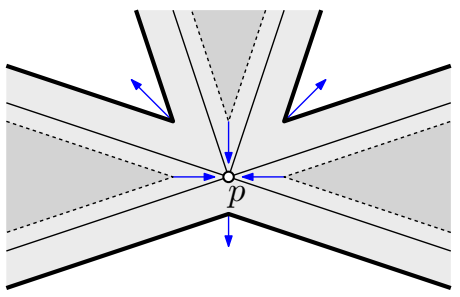

Fig. 2. A wavefront before (dotted), at (solid), and after an event (bold), with blue arrows showing movement direction of wavefront vertices. The standard pairing technique for handling a split event pairs each edge with its other neighbor in the cyclical order.

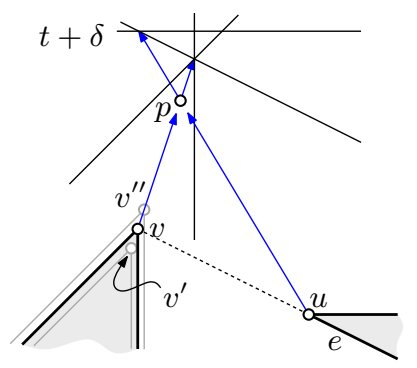

(a)

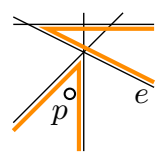

(b)

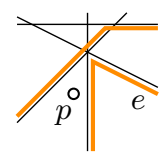

(c)
Fig. 3. Two wavefront vertices meet at $p$. There are two possibilities, (b) and (c), in order to pair up the edges such that the wavefront remains planar in a weak sense. of them leads to a crossing. The other two possibilities are illustrated in Fig. 3 (b, c). Both remaining possibilities are not planar in a strict sense. Still, there are no crossings - instead edges only touch. This shows there is no way to pair up the edges and remain strictlyplanar. 
Let us suppose that we initially move $v$ slightly away from $p$ or slightly closer to $p$. We obtain $v^{\prime}$ and $v^{\prime \prime}$ respectively, see Fig. 3 (a). We adapt their speeds such that they still reach $p$ at time $t$. Since $v^{\prime}$ moves slightly faster than $v$ and $e$, at time $t$, the vertex $v^{\prime}$ overtakes $e$. Similarly, $e$ overtakes $v^{\prime \prime}$. Hence, if we replace $v$ by $v^{\prime}$, the pairing in Fig. 3 (b) becomes invalid, and if we replace $v$ by $v^{\prime \prime}$, the pairing in Fig. 3 (c) becomes invalid. In particular, for the latter case the only valid pairing is the original pairing and the standard pairing technique fails.

For our further discussions it will be necessary to define precisely what we mean by event or weak planarity. Let $\Phi$ denote the set of all straight-line embeddings $\varphi: V \rightarrow \mathbb{R}^{2}$ of a graph $G=(V, E)$. The pair $\left(\Phi,\|\cdot\|_{\infty}\right)$ constitutes a normed space, where $\|.\|_{\infty}$ is defined by $\|\varphi\|_{\infty}=\max _{v \in V}\|\varphi(v)\|$. Note that the set of planar ${ }^{4}$ straight-line embeddings is an open subset of $\Phi$ w.r.t. the usual topology induced by $\|\cdot\|_{\infty}$.

Definition 1. The set of weakly-planar embeddings of $G$ is the topological closure of the set of planar embeddings of $G$.

This implies that every planar embedding is weakly-planar as well. In addition, for every weakly-planar embedding $\varphi$ and for every $\varepsilon>0$ there is a planar $\varepsilon$-perturbation $\varphi^{\prime}$ of $\varphi$, that is, $\left\|\varphi-\varphi^{\prime}\right\|_{\infty}<\varepsilon$. This definition allows us now to rephrase the fundamental principle as follows:

\section{At all times, the wavefront is a weakly-planar collection of polygons.}

Events. The wavefront $\mathcal{W}$ is initially weakly-planar. Informally, an event occurs when the wavefront is about to cease being weakly-planar and event handling needs to restructure the wavefront locally such that it can continue propagating in a weakly-planar fashion.

Assume that $\mathcal{W}\left(t^{\prime}\right)$ remains weakly-planar for all $t^{\prime} \in[t-\delta, t]$ and some $\delta>0$. For this time interval, we can consider $\mathcal{W}$ to be a kinetic planar straightline graph with a fixed set of kinetic vertices and edges. For Definition 2, we fix the vertex and edge set of $\mathcal{W}$, including the velocities of the vertices and temporarily ignore event handling. Furthermore, we denote by $B(p, r)$ the closed disk centered at $p$ with radius $r$ and by $\mathcal{W}\left(t^{\prime}\right) \cap B(p, r)$ the planar straight-line graph $\mathcal{W}\left(t^{\prime}\right)$ with all edges truncated to fit into $B(p, r)$ or removed if they entirely reside outside $B(p, r)$.

Definition 2. At location $p$ and time $t$ an event happens if at least two vertices meet at time $t$ at $p$ or if $\exists \varepsilon_{0}>0 \forall \varepsilon \in\left(0, \varepsilon_{0}\right) \exists \delta>0$ such that

(i) $\mathcal{W}\left(t^{\prime}\right) \cap B(p, \varepsilon)$ is non-empty and weakly-planar for $t^{\prime} \in[t-\delta, t]$ and

(ii) $\mathcal{W}\left(t^{\prime}\right) \cap B(p, \varepsilon)$ is non-empty and not weakly-planar for $t^{\prime} \in(t, t+\delta]$.

We call the edges that meet $p$ at time the edges which are involved in the event.

\footnotetext{
${ }^{4}$ A straight-line embedding $\varphi$ is called planar if its edges do not intersect except at common endpoints.
} 
As this definition defines events localized at some point $p$, we can also talk about multiple events occurring at the same time $t$ at different locations. If an event happens at location $p$ and time $t$ then, typically, weak planarity of $\mathcal{W}$ is violated locally around $p$ after time $t$. However, weak planarity is not violated if, for instance, a wavefront polygon collapses to a point. Fig. 3 gives another example where weak planarity is not violated after the event. The goal of event handling is to restore weak planarity by locally adapting the wavefront structure. We also want to remark that in certain cases multiple ways to correctly handle an event may exist, where one solution yields only a weakly-planar wavefronts while a different one produces a strictly-planar wavefront after the event.

Definition 3. We call the event at location $p$ and time $t$ elementary if three edges are involved. We call it an edge event if $B(p, \varepsilon) \backslash \mathcal{W}(t-\delta)$ consists of two connected components and a split event otherwise. Non-elementary edge and split events are called multi-edge and multi-split events respectively.

It is known how to handle edge events and elementary split events [4]. In the following, we present one unified approach that is able to correctly handle any type of event, including, in particular, multi-split events. Consequently, one side effect of our definition of weighted straight skeletons is that the distinction between edge events and split events becomes unnecessary.

\subsection{Pairing edges}

Assume an event happens at time $t$ at location $p$. Up until time $t$ the wavefront $\mathcal{W}$ is weakly-planar, and it becomes not weakly-planar after $t$. In order to restore weak planarity, we have to transform the wavefront structure. This involves repairing of wavefront edges.

We reduce the problem of pairing up wavefront edges during event handling to a particular matching problem, discussed in Section 3. This problem, which we study independently of straight skeletons, takes a pseudo-line arrangement in general position as input and provides us with a means to construct a weakly-planar wavefront again. In the following, we describe how to transform a weakly-planar wavefront into a suitable pseudo-line arrangement for the matching problem.

The pseudo-lines stem from the supporting lines of wavefront edges and are required to be in general position. By general position we mean that any pair of lines intersect in exactly one unique point. In particular, this implies that no two lines are parallel, no two lines are identical, and no three lines intersect in a common point.

At time $t$, several edges of the wavefront $\mathcal{W}$ are incident at location $p$. For each such edge, either zero, one, or both endpoints approach $p$ at time $t$. We construct a simplified version of the wavefront, denoted by $\mathcal{W}^{\prime}$, by dropping edges where both endpoints reach $p$ and joining its two endpoints. Furthermore, any edge where no endpoint reaches $p$ is split into two edges by a new wavefront vertex that also reaches $p$ at time $t$. Thus, in $\mathcal{W}^{\prime}$ an even number of wavefront edges have exactly one endpoint at point $p$ at time $t$, see Fig. 4 . 
Next, we choose $\varepsilon$ and $\delta$ sufficiently small, such that no other event happens between $t$ and $t+\delta$ and that exactly the edges involved in the event intersect $B(p, \varepsilon)$ during the interval $[t, t+\delta]$. We obtain $\mathcal{W}^{\prime \prime}$ from $\mathcal{W}^{\prime}$ by perturbing the locations of its vertices. This perturbation shall satisfy the following properties: (i) The edges involved in the event still reach $p$ at time $t$. (ii) The supporting lines of involved edges are in general position at time $t+\delta$. (iii) The perturbation is such that $\mathcal{W}^{\prime \prime}$ is strictly-planar everywhere outside $B(p, \varepsilon)$ at time $t+\delta$. (iv) The perturbation is such that any vertex is (at time $t+\delta$ ) on the same side of the supporting line through any edge in both $\mathcal{W}^{\prime}$ and $\mathcal{W}^{\prime \prime}$. The set of supporting lines at time $t+\delta$ then shall be the input to the matching problem.

We use the new pairing obtained from the matching algorithm to construct a new (still perturbed) wavefront $\mathcal{W}^{\prime \prime \prime}$ from $\mathcal{W}^{\prime \prime}$. The new pairing ensures that $\mathcal{W}^{\prime \prime \prime}$ is strictly-planar around $p$ after time $t$, see Lemma 7 in Section 3.3. If several multi-split events happen at the same time, then this procedure is repeated for every such event independently. Each event will locally restore strict planarity, and, thus, global strict planarity will be restored. Finally, we revert the perturbation on $\mathcal{W}^{\prime \prime \prime}$ and obtain the new post-event wavefront.

Lemma 1. The new post-event wavefront $\mathcal{W}^{\star}$ is weakly-planar.

Proof. Note that the perturbation we apply to obtain $\mathcal{W}^{\prime \prime}$ from $\mathcal{W}^{\prime}$ was sufficiently small such that no vertex could "jump" over the supporting line of any edge of the wavefront. Therefore, if we assume to the contrary that $\mathcal{W}^{\star}$ is not weakly-planar, that would imply that the perturbed wavefront $\mathcal{W}^{\prime \prime \prime}$ was not (strictly) planar either. Since $\mathcal{W}^{\prime \prime \prime}$ is (strictly) planar outside of $B(p, \varepsilon)$ per our requirement for the perturbation and is (strictly) planar within $B(p, \varepsilon)$ due to the new pairing, this is a contradiction.

\section{Matchings and roommates}

For an even $N$, let $\mathcal{L}=\left\{\ell_{1}, \ldots, \ell_{N}\right\}$ be an oriented pseudo-line arrangement in general position, i.e., a set of directed Jordan-curves that begin and end at infinity and intersect each other in single, unique points. Let $\mathcal{C}$ be a pseudo-circle that encloses all intersections of pseudo-lines and that intersects each (directed) pseudo-line $\ell$ exactly twice, once in its begin-point $b(\ell)$ and once in its end-point.

A matching $M$ in $\mathcal{L}$ is a grouping of $\ell_{1}, \ldots, \ell_{N}$ into pairs. The matching tail of $\ell_{i}$ is the sub-curve of $\ell_{i}$ from $b\left(\ell_{i}\right)$ to $\ell_{i} \times M\left(\ell_{i}\right)$, i.e., the point where $\ell_{i}$ intersects its matching-partner $M\left(\ell_{i}\right)$. 
Definition 4. A matching in $\mathcal{L}$ is called planar if the union of the matching tails gives a planar drawing.

The planar matching problem is the problem of finding a planar matching $M$ for a given pseudo-line arrangement $\mathcal{L}$ in general position. In the following we translate the planar matching problem into a stable roommate problem.

\subsection{The stable roommate problem}

Assume that we have an even number $N$ of elements $\mathcal{A}=\left\{a_{1}, \ldots, a_{N}\right\}$. Each element has a ranking of elements, which is complete and strict, i.e., all elements are ranked and no two elements are ranked the same. Let $M$ be a matching of $a_{1}, \ldots, a_{N}$. A pair $\left\{a_{i}, a_{j}\right\}$ is a blocking pair for $M$ if $a_{i}$ prefers $a_{j}$ over $M\left(a_{i}\right)$ and $a_{j}$ prefers $a_{i}$ over $M\left(a_{j}\right)$. A matching is stable if there is no blocking pair. The stable roommate problem asks for a stable matching in $\mathcal{A}$. The stable roommate problem is a well-studied problem in optimization theory (see, for example, Fleiner et al. [6] and the references therein). In particular, not every instance of the stable roommate problem has a solution, and testing whether it has a solution can be done in polynomial time.

Let us again consider the directed pseudo-line arrangement $\mathcal{L}$. As we walk along a pseudo-line $\ell_{i}$ from its begin-point to its end-point we encounter all other pseudo-lines in $\mathcal{L}$. This order naturally gives us a complete and strict ranking for $\ell_{i}$ if we attach $\ell_{i}$ itself at the end of the list. Thus, $\mathcal{L}$ defines an instance of the stable roommate problem.

Lemma 2. A directed pseudo-line arrangement has a planar matching if and only if the corresponding stable roommate instance has a stable matching.

Proof. For a matching $M$, the matching tails of two pseudo-lines $\ell_{i}, \ell_{j}$ cross if and only if $\ell_{i}$ prefers $\ell_{j}$ over $M\left(\ell_{i}\right)$ and $\ell_{j}$ prefers $\ell_{i}$ over $M\left(\ell_{j}\right)$. Hence, the matching is non-planar if and only if there is a blocking pair.

\section{$3.2 \quad$ Stable partitions}

In order to solve our particular stable roommate problem, we review some results on so-called stable partitions, mostly based on a paper by Tan and Hsueh [12].

Let $\mathcal{A}$ be an instance of a stable roommate problem, and let $\pi$ be a permutation on $\mathcal{A}$, i.e., a bijective map $\mathcal{A} \rightarrow \mathcal{A}$. This function partitions $\mathcal{A}$ into one or more cycles, i.e., sequences $a_{0}^{\prime}, \ldots, a_{k-1}^{\prime}$ in $\mathcal{A}$ with $a_{0}^{\prime} \stackrel{\pi}{\rightarrow} a_{1}^{\prime} \stackrel{\pi}{\rightarrow} \ldots \stackrel{\pi}{\rightarrow} a_{k-1}^{\prime} \stackrel{\pi}{\rightarrow} a_{0}^{\prime}$. A cycle with $k \geq 3$ is called a semi-party cycle if $a_{i}^{\prime}$ prefers $\pi\left(a_{i}^{\prime}\right)$ over $\pi^{-1}\left(a_{i}^{\prime}\right)$. A semi-party partition of $\mathcal{A}$ is a permutation of $\mathcal{A}$ where all cycles with $k \geq 3$ are semi-party cycles.

Given a semi-party partition $\pi$, a pair $\left\{a_{i}, a_{j}\right\}$ is called a party-blocking pair if $a_{i}$ prefers $a_{j}$ over $\pi^{-1}\left(a_{i}\right)$ and $a_{j}$ prefers $a_{i}$ over $\pi^{-1}\left(a_{j}\right)$. A stable partition is a semi-party partition that has no party-blocking pairs. The cycles of a stable partition are called parties. An odd (even) party is a party of odd (even) cardinality. Furthermore, $a_{i}, a_{j}$ are party-partners if $a_{i}=\pi\left(a_{j}\right)$ or $a_{j}=\pi\left(a_{i}\right)$. 
Theorem $1([\mathbf{1 1}, \mathbf{1 2}])$. For any instance $\mathcal{A}$ of the stable roommate problem the following statements hold:

1. $\mathcal{A}$ has a stable partition, and it can be found in polynomial time.

2. Any stable partition of $\mathcal{A}$ has the same number of odd parties.

3. $\mathcal{A}$ has a stable matching if and only if it has a stable partition with no odd parties.

\subsection{Existence of planar matchings}

Now we consider parties that occur in stable roommate instances defined by a directed pseudo-line arrangement $\mathcal{L}$. Theorem 1(1) gives us a stable partition $\pi$ for $\mathcal{L}$. Let a singleton-party, a pair-party, and a cycle-party be a party consisting of one, two, and at least three pseudo-lines, respectively. For all pseudo-lines $\ell$ that are not a singleton-party, let their party-tail be the part between $b(\ell)$ and $\ell \times \pi^{-1}(\ell)$. For any pseudo-line $\ell$ that is a singleton-party, let its party-tail be the part of $\ell$ between begin-point and end-point.

Lemma 3. The party-tails of two pseudo-lines $\ell, \ell^{\prime}$ do not intersect unless $\ell$ and $\ell^{\prime}$ are party-partners.

Proof. Assume that $\ell \times \ell^{\prime}$ belongs to both party-tails, but $\ell$ and $\ell^{\prime}$ are not partypartners. We first show that $\ell$ prefers $\ell^{\prime}$ over $\pi^{-1}(\ell)$. This holds automatically if $\ell$ is a singleton-party, because then $\pi^{-1}(\ell)=\ell$, and any pseudo-line ranks itself lowest. If $\ell$ is not a singleton-party, then the party-tail of $\ell$ consists of the sub-curve between $b(\ell)$ and $\ell \times \pi^{-1}(\ell)$. Since $\ell^{\prime} \neq \pi^{-1}(\ell)$ by assumption, and since no three pseudo-lines intersect in a point, $\ell \times \ell^{\prime}$ comes strictly earlier than $\ell \times \pi^{-1}(\ell)$ when walking along $\ell$. By definition of the ranking for directed pseudo-lines, hence $\ell$ prefers $\ell^{\prime}$ over $\pi^{-1}(\ell)$.

Similarly one shows that $\ell^{\prime}$ prefers $\ell$ over $\pi^{-1}\left(\ell^{\prime}\right)$. Hence, $\left\{\ell, \ell^{\prime}\right\}$ is a partyblocking pair and $\pi$ is not a stable partition, a contradiction.

Lemma 4. There cannot be two singleton-parties.

Proof. Assume that $P$ and $P^{\prime}$ are two singleton-parties, with $P=\{\ell\}$ and $P^{\prime}=\left\{\ell^{\prime}\right\}$. Since they are singleton-parties, their party-tails extend from their begin-points to their end-points. Since all pseudo-lines intersect within $\mathcal{C}$, so do $\ell$ and $\ell^{\prime}$. But $\ell$ and $\ell^{\prime}$ are not party-partners, in contradiction to Lemma 3.

Lemma 5. There cannot be two cycle-parties.

Proof. Assume we have two cycle-parties $P_{1}=\left\{\ell_{0}, \ell_{1}, \ldots, \ell_{a-1}\right\}$ and $P_{2}=$ $\left\{\ell_{0}^{\prime}, \ell_{1}^{\prime}, \ldots, \ell_{b-1}^{\prime}\right\}$, with $\pi\left(\ell_{i}\right)=\ell_{i+1}$, addition modulo $a$, and $\pi\left(\ell_{i}^{\prime}\right)=\ell_{i+1}^{\prime}$ with addition modulo $b$.

Let $G\left(P_{1}\right)$ be the graph formed by the party tails of $P_{1}$ as follows: The vertex set comprises $b(\ell)$ and $\ell \times \pi(\ell)$ for every pseudo-line $\ell$ in $P_{1}$. We add each party-tail as two edges $(b(\ell), \ell \times \pi(\ell))$ and $\left(\ell \times \pi(\ell), \pi^{-1}(\ell) \times \ell\right)$, see Fig. 5 . 
Note that $G\left(P_{1}\right)$ has the following structure: It consists of a cycle $\mathcal{C}_{1}$ of edges of the form $\left(\ell \times \pi(\ell), \pi^{-1}(\ell) \times \ell\right)$ together with one edge attached to each vertex of $\mathcal{C}_{1}$ of the form $(b(\ell), \ell \times \pi(\ell))$. By Lemma $3, G\left(P_{1}\right)$ is planar. Note that the vertices $b\left(\ell_{0}\right), b\left(\ell_{1}\right), \ldots, b\left(\ell_{a-1}\right)$ lie on $\mathcal{C}$ and are ordered clockwise or counterclockwise. Therefore, $G\left(P_{1}\right)$ tessellates the area enclosed by $\mathcal{C}$ into $a+1$ regions. Note that exactly $a$ of those regions are partially bounded by $\mathcal{C}$. The remaining region is the one bounded by $\mathcal{C}_{1}$. Similarly, we define $G\left(P_{2}\right)$ and $\mathcal{C}_{2}$.

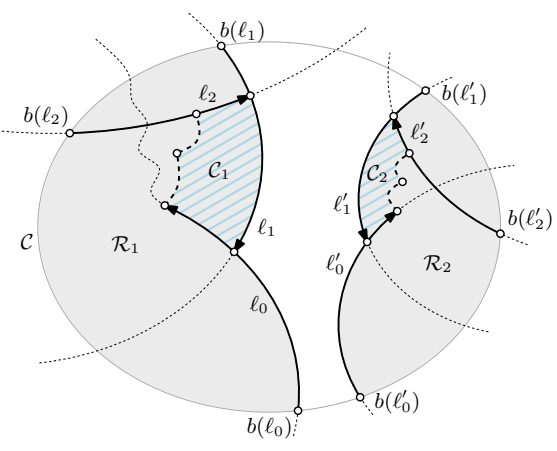

Fig. 5. The two pseudo-lines $\ell_{0}$ and $\ell_{0}^{\prime}$ cannot intersect.

Again by Lemma 3, $G\left(P_{1}\right) \cup G\left(P_{2}\right)$ is planar, and it follows that $G\left(P_{2}\right)$ is entirely contained in one region of $G\left(P_{1}\right)$. This region is not the region bounded by $\mathcal{C}_{1}$. We denote by $\mathcal{R}_{1}$ the union of all regions of $G\left(P_{1}\right)$ that do not contain $G\left(P_{2}\right)$. Likewise, we denote by $\mathcal{R}_{2}$ the union of all regions of $G\left(P_{2}\right)$ that do not contain $G\left(P_{1}\right)$. We observe that $\mathcal{R}_{1}$ and $\mathcal{R}_{2}$ are disjoint.

Without loss of generality, the boundary of $\mathcal{R}_{1}$ consists of parts of $\mathcal{C}$ as well as the path $b\left(\ell_{1}\right), \ell_{1} \times \ell_{2}, \ell_{0} \times \ell_{1}, b\left(\ell_{0}\right)$. Likewise, $\mathcal{R}_{2}$ is bounded by parts of $\mathcal{C}$ and edges stemming from $\ell_{1}^{\prime}$ and $\ell_{0}^{\prime}$.

In the following, we will show that $\ell_{0}$ cannot intersect $\mathcal{R}_{2}$, and, conversely, $\ell_{0}^{\prime}$ cannot intersect $\mathcal{R}_{1}$. Consequently, $\ell_{0}$ does not intersect $\ell_{0}^{\prime}$ within $\mathcal{C}$, which is a contraction as we require each pair of pseudo-lines to intersect exactly once in the area enclosed by $\mathcal{C}$. This concludes the proof.

Note that $\ell_{0}$ starts at $b\left(\ell_{0}\right)$, then makes up parts of the boundary of $\mathcal{R}_{1}$ until it reaches $\ell_{0} \times \ell_{1}$. Then, $\ell_{0}$ moves into the interior of $\mathcal{R}_{1}$ as it makes up an edge of $\mathcal{C}_{1}$, but not the one that is part of the boundary of $\mathcal{R}_{1}$. Once $\ell_{0}$ enters $\mathcal{R}_{1}$, it can leave only by intersecting $\mathcal{C}$ at its end-point as it is not allowed to self-intersect or to intersect $\ell_{1}$ a second time. After $\ell_{0}$ has left the area enclosed by $\mathcal{C}$, it cannot enter again, as it intersects $\mathcal{C}$ exactly twice. Likewise, $\ell_{0}^{\prime}$ will exit the area enclosed by $\mathcal{C}$ through its end-point in $\mathcal{R}_{2}$ and cannot intersect $\mathcal{R}_{1}$.

Lemma 6. There cannot be a singleton-party and a cycle-party.

Proof. We follow the same idea as in the previous proof, and use $P_{1}$ as the cycleparty and $P_{2}$ as the singleton-party. Let $\ell_{0}$ be defined as previously, and use $\ell_{0}^{\prime}$ as the single line in $P_{2}$. Since the tail of $\ell_{0}^{\prime}$ consists of all points between the begin-point and the end-point of $\ell_{0}^{\prime}$, again no intersection between $\ell_{0}$ and $\ell_{0}^{\prime}$ is possible.

Theorem 2. No instance of a stable roommate problem defined by a directed pseudo-line arrangement $\mathcal{L}$ can have an odd party.

Proof. Assume to the contrary that some stable partition $\pi$ has an odd party $P$. As $\mathcal{L}$ comprises an even number of pseudo-lines, there needs to be another 

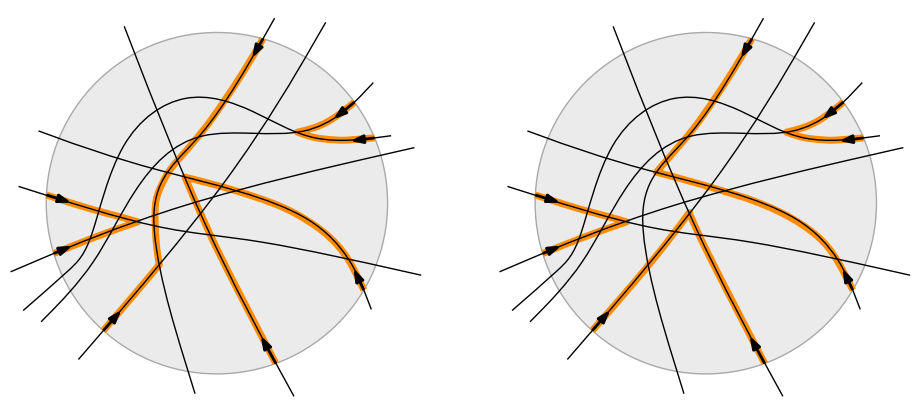

Fig. 6. A planar matching of pseudo-lines specifies how to construct a weakly-planar post-event wavefront. One arrangement may have multiple planar matchings.

odd party $P^{\prime}$. This can only happen if there are two singleton-parties, two (odd) cycle parties, or a singleton-party and an (odd) cycle-party. These are ruled out by Lemma 4, Lemma 5, and Lemma 6, respectively.

Theorem 3. Every directed pseudo-line arrangement has a planar matching, and it can be found in polynomial time.

Proof. This is a direct result of Lemma 2, Theorem 1, and Theorem 2.

By Theorem 1 we can find a stable partition in polynomial time. By Theorem 2 and Lemma 5, it consists of pair-parties, except for at most one cycle-party $P$ that has even length. If there is no cycle-party, then the stable partition is in fact a stable matching. Otherwise, if say $\ell_{1}, \ldots, \ell_{N}$ is the even cycle-party, then we can find stable matching $M$ easily, and there are two choices: Either set $M\left(\ell_{2 i}\right)=\pi\left(\ell_{2 i}\right)$ and $M\left(\ell_{2 i+1}\right)=\pi^{-1}\left(\ell_{2 i+1}\right)$, or do the same after shifting all indices by one.

\subsection{Application to straight skeletons}

The matching tails of the pseudo-lines play the role of wavefront edges after the event. The matching tells us how to pair up the wavefront edges in order to restore planarity locally at $p$, see Fig. 6 .

Lemma 7. There exists a weakly-planar wavefront after the event if there is a planar matching for $\mathcal{L}$.

Using Theorem 3, we have found a stable matching and with it a weaklyplanar post-event wavefront, in polynomial time. Notice that if a cycle-party exists, then there are two possible post-event wavefronts. Consequently, ambiguities in the development of the wavefront may be caused by edge events between parallel edges [4] and multi-split events alike. 


\section{Conclusion}

Although algorithms and even rudimentary implementations to construct the weighted straight skeleton were previously presented, and even though several applications are suggested in the literature, this paper is the first to provide a concrete, constructive proof that a well-defined weighted straight skeleton actually exists in all cases. This result is based on two main ingredients: First, we introduced and studied planar matchings on a directed pseudo-line arrangement as a generic tool independent of straight skeletons. In particular, we showed that planar matchings always exist. Second, our interpretation of an event as violation of (weak) planarity unifies the classification of edge and split events in $2 \mathrm{D}$ and promises to simplify the description and study of straight skeletons in dimensions higher than two, where the number of types and complexity of events would significantly increase otherwise.

Acknowledgments. We would like to thank David Eppstein for the idea of interpreting the edge-pairing problem as a stable roommate problem.

\section{References}

1. O. Aichholzer, D. Alberts, F. Aurenhammer, and B. Gärtner. Straight Skeletons of Simple Polygons. In Proc. 4th Int'l Symp. of LIESMARS, pages 114-124, 1995.

2. F. Aurenhammer. Weighted Skeletons and Fixed-Share Decomposition. Comput. Geom. Theory and Appl., 40(2):93-101, July 2008.

3. G. Barequet, D. Eppstein, M. T. Goodrich, and A. Vaxman. Straight Skeletons of Three-Dimensional Polyhedra. In Proc. 16th Annu. Europ. Symp. Algorithms, pages 148-160, Sept. 2008.

4. T. Biedl, M. Held, S. Huber, D. Kaaser, and P. Palfrader. Weighted Straight Skeletons in the Plane. Comput. Geom. Theory and Appl., 48(2):120-133, Feb. 2015.

5. D. Eppstein and J. Erickson. Raising Roofs, Crashing Cycles, and Playing Pool: Applications of a Data Structure for Finding Pairwise Interactions. Discrete Comput. Geom., 22(4):569-592, 1999.

6. T. Fleiner, R. W. Irving, and D. F. Manlove. Efficient algorithms for generalized stable marriage and roommates problems. Theoretical Computer Science, 381(13):162 - 176, 2007.

7. J.-H. Haunert and M. Sester. Area Collapse and Road Centerlines Based on Straight Skeletons. GeoInformatica, 12:169-191, 2008.

8. S. Huber. Computing Straight Skeletons and Motorcycle Graphs: Theory and Practice. Shaker Verlag, Apr. 2012. ISBN 978-3-8440-0938-5.

9. T. Kelly and P. Wonka. Interactive Architectural Modeling with Procedural Extrusions. ACM Trans. Graph., 30(2):14:1-14:15, Apr. 2011.

10. R. Laycock and A. Day. Automatically Generating Large Urban Environments Based on the Footprint Data of Buildings. In Proc. 8th Symp. Solid Modeling Applications, pages 346-351, June 2003.

11. J. J. Tan. A Necessary and Sufficient Condition for the Existence of a Complete Stable Matching. Journal of Algorithms, 12(1):154-178, Mar. 1991.

12. J. J. Tan and Y.-C. Hsueh. A generalization of the stable matching problem. Discrete Applied Mathematics, 59(1):87-102, 1995. 